Research Article

\title{
Effects of SMILE Surgery on Intraocular Pressure, Central Corneal Thickness, Axial Length, Peripapillary Retinal Nerve Fiber Layer, and Macular Ganglion Cell Complex Thickness
}

\author{
Zhanlin Zhao, ${ }^{1,2,3,4,5}$ Sylvain Michée, ${ }^{1,6}$ Jean-François Faure, ${ }^{6}$ Christophe Baudouin, ${ }^{1,2,3}$ \\ and Antoine Labbé $\mathbb{D}^{1,2,3}$ \\ ${ }^{1}$ Department of Ophthalmology, Ambroise Paré Hospital, IHU FOReSIGHT, AP-HP, UFR Paris-île de France Ouest, \\ University of Paris Saclay, Boulogne-Billancourt, France \\ ${ }^{2}$ Department of Ophthalmology III, Quinze-Vingts Hospital, IHU FOReSIGHT, Paris, France \\ ${ }^{3}$ CHNO des Quinze-Vingts, IHU FOReSIGHT, Inserm-DGOS CIC 1423, 17 Rue Moreau, Paris 75012, France \\ ${ }^{4}$ Department of Ophthalmology, Ninth People's Hospital, Shanghai JiaoTong University School of Medicine, Shanghai, China \\ ${ }^{5}$ Shanghai Key Laboratory of Orbital Diseases and Ocular Oncology, Shanghai, China \\ ${ }^{6}$ Espace Nouvelle Vision, Ophthalmological Clinic, Paris, France
}

Correspondence should be addressed to Antoine Labbé; alabbe@15-20.fr

Received 18 May 2020; Revised 4 November 2020; Accepted 18 November 2020; Published 29 November 2020

Academic Editor: Biju B. Thomas

Copyright $(2020$ Zhanlin Zhao et al. This is an open access article distributed under the Creative Commons Attribution License, which permits unrestricted use, distribution, and reproduction in any medium, provided the original work is properly cited.

Purpose. To evaluate the change in intraocular pressure (IOP), central corneal thickness (CCT), axial length, peripapillary retinal nerve fiber layer (RNFL) thickness, and macular ganglion cell complex (GCC) thickness after small incision lenticule extraction (SMILE) surgery. Methods. This prospective observational study was conducted in Espace Nouvelle Vision, Ophthalmological Clinic, Paris, France. Fifty eyes of 25 patients were enrolled in this study and underwent SMILE surgeries. IOP, central corneal thickness (CCT), axial length (AL), peripapillary RNFL thickness, and macular GCC thickness were measured before and at 3 months after SMILE. Results. The mean preoperative spherical equivalent was $-3.15 \pm 1.50$ diopters (D), and the mean postoperative value was $0.15 \pm 0.28 \mathrm{D}$. After SMILE surgery, IOP decreased from $15.03 \pm 2.79 \mathrm{mmHg}$ to $11.02 \pm 2.73 \mathrm{mmHg}$ and $10.02 \pm 2.21 \mathrm{mmHg}$ at 1 and 3 months, respectively $(P<0.01$ for both comparisons). The mean decrease in measured IOP as a function of ablation depth was $0.065 \pm 0.031 \mathrm{mmHg} / \mu \mathrm{m}$. CCT decreased from $545.98 \pm 26.61 \mu \mathrm{m}$ to $478.40 \pm 30.26 \mu \mathrm{m}$ after SMILE surgery $(P<0.01)$. AL decreased from $24.80 \pm 0.84 \mathrm{~mm}$ to $24.70 \pm 0.83 \mathrm{~mm}(P<0.01)$. There was no statistically significant change in mean peripapillary RNFL or mean GCC thickness after SMILE surgery. Conclusions. SMILE surgery modified IOP measurement, CCT, and AL but did not change peripapillary RNFL and macular GCC thicknesses. The postoperative drop in measured IOP might be explained by the decreased CCT. An accurate re-evaluation of AL should be performed before cataract surgery among post-SMILE patients.

\section{Introduction}

Small incision lenticule extraction (SMILE) is a novel form of refractive surgery for the treatment of myopia and myopic astigmatism. With the development of the femtosecond laser in 2006, SMILE has become the newest refractive laser procedure, after photorefractive keratectomy (PRK) and femtosecond-assisted laser in situ keratomileusis (FS-LASIK) $[1,2]$. During the SMILE procedure, an intrastromal lenticule is created using the femtosecond lenticule extraction (FLEx) technique $[3,4]$. Then, the lenticular interfaces are separated by a dissector through a small $2-3 \mathrm{~mm}$ incision so that the lenticule can be removed in a flapless fashion. With the advantages of better ocular surface stability and biomechanical strength, SMILE is gaining worldwide acceptance and increasing popularity in recent years. Therefore, its effects on eyes, particularly on IOP, and, consequently, the retinal nerve fiber layer should be fully understood. 
Intraocular pressure (IOP) has been reported to increase during both FS-LASIK $[5,6]$ and SMILE refractive surgeries $[7,8]$. A transient elevated IOP up to $65-80 \mathrm{mmHg}$ has been reported during FS-LASIK flap creation using the femtosecond laser [6]. Intraoperative and postoperative complications such as macular hole [9] and retinal detachment [10] related to the sudden increase in IOP have been reported in FS-LASIK patients. In addition, previous studies have shown mild, reversible, and localized macular edema and thinning of the retinal nerve fiber layer (RNFL) soon after FS-LASIK in adults [11-13]. During the SMILE procedure, a curved lens is applied to the edge of the cornea and limbus, and the suction pressure generated by the VisuMax laser system is lower than in FS-LASIK, approximately $35 \mathrm{mmHg}$ [14]; consequently, SMILE should not induce complications related to a sudden increase in IOP, as the pressure is low enough to maintain the intraocular circulation. However, the FLEx procedure takes significantly more time (25-28 seconds) than FS-LASIK; therefore, eyes undergoing FLEx are exposed to a longer duration of increased IOP [8].

In addition, measured IOP has been reported to decrease both after FS-LASIK and SMILE surgeries, as refractive surgeries flatten the corneal profile [15]. IOP seems to remain more stable after SMILE than FS-LASIK [15]. As a flapless technique, SMILE might have less influence on corneal biomechanical stability as compared to FS-LASIK [16]. Given the frequency of refractive surgeries and the incidence of glaucoma in the general population [17], the risk of glaucoma in patients undergoing refractive surgeries and the intraoperative impact of the SMILE procedure on optic nerve fibers, IOP, and CCT should be evaluated. Thus, the purpose of this study was to evaluate the effect of SMILE surgery on IOP, CCT, axial length, peripapillary retinal nerve fiber layer (RNFL) thickness, and macular ganglion cell complex (GCC) thickness.

\section{Methods}

This prospective observational study included 50 eyes of 25 consecutive patients (29.42 \pm 4.47 years of age) undergoing SMILE between November 2018 and July 2019 at the Espace Nouvelle Vision in Paris, France. All patients were informed of the purpose of the study, and their consent was obtained according to the Declaration of Helsinki. Inclusion criteria were the standard SMILE criteria: age less than 60 years, history of stable refractive error, minimum corneal thickness of $500 \mu \mathrm{m}$, minimum residual bed of $250 \mu \mathrm{m}$, and regular topography as determined by the ATLAS 9000 corneal topography system (Zeiss, Germany).

Exclusion criteria were as follows: previous ocular refractive surgeries; presence of glaucoma; IOP higher than $21 \mathrm{mmHg}$; evidence of glaucomatous optic nerve damage; presence of severe dry eye, progressive corneal degeneration, keratoconus or corneal scars; pregnancy or breast feeding; or presence of diseases that affect the regenerative process of the cornea (diabetes mellitus and collagen-related disease). Preoperative examination included refraction, IOP measurement, topography, slit-lamp biomicroscopy, and optic nerve head and macular OCT scanning. At each visit,
logMAR uncorrected distance visual acuity (UDVA) and manifest refraction were also recorded.

2.1. IOP and CCT Measurement. IOP and CCT were measured before and at 1 month and 3 months after surgery using a noncontact tono/pachymeter (NT-530P, NIDEK). The tono/pachymeter was calibrated according to the manufacturer's guidelines prior to its use in this study. At least three measurements of IOP without CCT correction were obtained for each patient. The means of those measurements were noted for analysis.

2.2. Axial Length Measurement. Axial length was measured using the IOLMaster 500 (Zeiss, Germany) before and 3 months after SMILE surgery. At least three measurements with signal-to-noise ratios of greater than 2.0 were obtained for each patient so as to minimize positional inaccuracies or repeatability issues. The means of those 3 measurements were recorded for analysis.

2.3. Peripapillary RNFL and Macular GCC Complex Measurements. All patients underwent OCT scanning using the Zeiss Cirrus HD-OCT device before and 3 months after surgery. Peripapillary RNFL was measured using the glaucoma analysis mode of the Cirrus SD OCT device (model 4000, software version 6.0, Carl Zeiss Meditec, Inc.). The optic nerve head was automatically scanned over an area of $6 \times 6 \mathrm{~mm}$ at $200 \times 200$ pixels resolution. The peripapillary RNFL thickness within the circular maps in each quadrant (superior, inferior, temporal, and nasal quadrants) was recorded for each patient. The ganglion cell analysis algorithm of the Cirrus SD OCT was used to process and measure the thickness of the macular ganglion cell layer (GCL) and inner plexiform layer (IPL). The mean and minimum GCL thicknesses were measured from the elliptical annulus centered on the fovea. Representative images were selected for analysis by considering criteria such as best focus, good contrast, and least motion artifacts with signal strength above seven. RNFL and GCL segmentation were verified for every OCT image.

2.4. Surgical SMILE Procedure. All procedures were performed under topical anesthesia (preservative-free benoxinate hydrochloride $0.4 \%$ eye drops) in all cases. The VisuMax (Carl Zeiss Meditec AG) femtosecond laser system was used to perform the surgical refractive corrections for patients using the SMILE protocol, with a repetition rate of $500 \mathrm{kHz}$ and a pulse energy of $130 \mathrm{~nJ}$. The laser created a peripheral corneal incision of $2.2 \mathrm{~mm}$ at $135^{\circ}$ at depths from 120 to $140 \mu \mathrm{m}$. The cap diameter was 6.9 to $7.6 \mathrm{~mm}$, and the optical zone varied from 5.9 to $6.6 \mathrm{~mm}$. Special SMILE forceps were used to enter the incision and remove the intrastromal lenticule. All surgical procedures were performed by a single surgeon (Dr. SM). An aspirating speculum (no 15961, Geuder, Heidelberg, Germany) was used to keep the eye open. 
2.5. Statistical Analysis. The one-way repeated measures ANOVA test was used for comparisons of IOP and CCT before and at 1 month and 3 months after surgery. Pearson correlation analyses were calculated between data using adjusted $R$ [2]. A $P$ value $<0.05$ (paired $t$-test, GraphPad Prism version 6.00 for Mac) was considered statistically significant for comparisons among preoperative and 3month postoperative RNFL and GCC data. Data were expressed as mean \pm standard error (SD).

\section{Results}

3.1. Clinical Parameters. Fifty eyes of 25 consecutive patients $(29.42 \pm 4.47$ years of age) undergoing bilateral SMILE were enrolled in this study. The mean preoperative spherical equivalent was $-3.15 \pm 1.50$ diopters (D), and the postoperative spherical equivalent was $0.15 \pm 0.28 \mathrm{D}$. The mean programmed ablation thickness was $81.24 \pm 24.76 \mu \mathrm{m}$. After SMILE surgery, IOP decreased from $15.03 \pm 2.79 \mathrm{mmHg}$ to $11.02 \pm 2.73 \mathrm{mmHg}$ and $10.02 \pm 2.21 \mathrm{mmHg}$ at 1 and 3 months, respectively $(P<0.01$ for both comparisons; Figure 1). The mean decrease in IOP at 3 months was $5.01 \pm 2.08 \mathrm{mmHg}$ as compared to preoperative data. The mean decrease in IOP as a function of ablation depth was $0.065 \pm 0.031 \mathrm{mmHg} / \mu \mathrm{m}$. CCT decreased from $545.98 \pm 26.61 \mu \mathrm{m}$ to $478.40 \pm 30.26 \mu \mathrm{m} 3$ months after SMILE surgery $(P<0.01)$. AL decreased from $24.80 \pm 0.84 \mathrm{~mm}$ (range: 23.12 to $26.27 \mathrm{~mm}$ ) to $24.70 \pm 0.83 \mathrm{~mm}$ (range: 23.02 to $26.14 \mathrm{~mm})(P<0.01)$. The clinical parameters are presented in Table 1.

3.2. Peripapillary RNFL Thickness Changes. Pre- and postoperative peripapillary RNFL values in the 4 quadrants are shown in Table 2 . The mean peripapillary RNFL thickness was $94.42 \pm 8.26 \mu \mathrm{m}$ prior to SMILE and $95.16 \pm 8.50 \mu \mathrm{m}$ at the third postoperative month $(P>0.05)$.

3.3. Macular GCL Thickness Changes. Pre- and postoperative mean and minimum values for GCL thickness are shown in Table 2. The mean macular GCL thickness was $78.08 \pm 11.00 \mu \mathrm{m}$ prior to SMILE and $79.78 \pm 6.22 \mu \mathrm{m}$ after 3 months $(P>0.05)$. The mean minimum GCL thickness was $78.54 \pm 5.08 \mu \mathrm{m}$ preoperatively and $79.22 \pm 5.14 \mu \mathrm{m}$ at 3 months postoperatively $(P>0.05)$. The difference was approximately $0.7 \mathrm{~mm}$, which is within the minimal resolution of OCT scans.

3.4. Correlation Analysis. Postoperative IOP values were significantly correlated with the preoperative IOP values $\left(R^{2}=0.46, P<0.01\right.$; Figure $\left.2(\mathrm{a})\right)$. The decrease in IOP was correlated with the spherical refractive changes $\left(R^{2}=0.1342\right.$, $P<0.01$; Figure 2(b)). Linear regression analysis showed that IOP decreased by $1.187 \mathrm{mmHg}$ for each diopter of spherical equivalent treated. No difference was observed in IOP values based on preoperative spherical refraction $\left(R^{2}=0.005428\right.$, $P=0.6111)$. There was a positive correlation between IOP changes and CCT changes $\left(R^{2}=0.1234, \quad P>0.05\right.$;

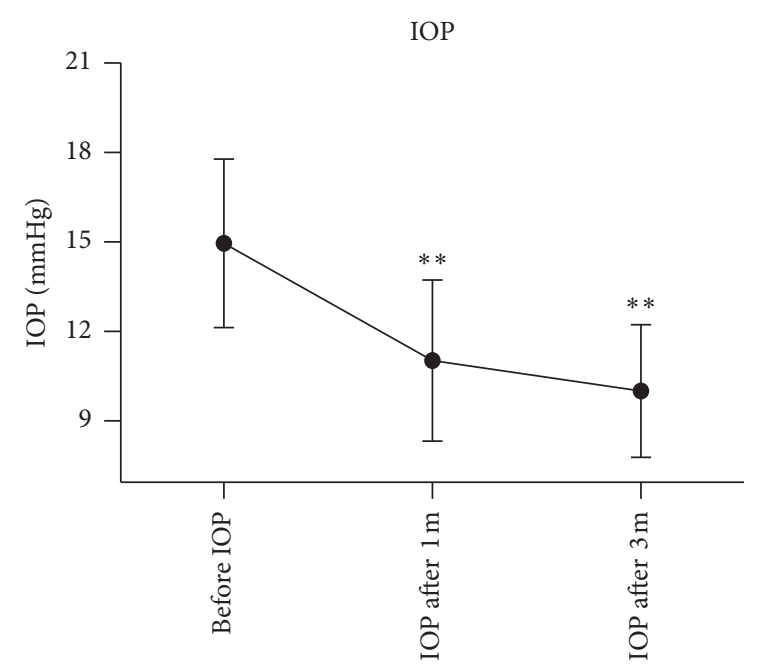

Figure 1: IOP decrease at 1 month and 3 months after SMILE. $F=142.8, P<0.01 ; * *$ compared with preoperative data, $P<0.01$.

Figure 2(c)). IOP tended to decrease more when CCT changed more $(\mathrm{IOP}=0.07083 * \mathrm{CCT})$. There was no correlation between IOP and axial length change $\left(R^{2}=0.04032\right.$, $P>0.05$; Figure 2(d)). There was a positive correlation between $\mathrm{AL}$ changes and programmed ablation thickness $\left(R^{2}=0.17, P<0.01\right.$; Figure $\left.2(\mathrm{e})\right)$ and a positive correlation between programmed ablation thickness and decreased CCT $\left(R^{2}=0.93, P<0.01\right.$; Figure 2(f)).

\section{Discussion}

SMILE is a new femtosecond laser-based flapless keratorefractive surgical procedure, taking a growing place among refractive surgeries to treat myopia and/or astigmatism. However, myopic patients are particularly at risk of developing open-angle glaucoma due to a variety of mechanisms, including increased susceptibility of the optic nerve head to damage by elevated IOP and the increased effect of shearing forces in optic nerve head damage $[18,19]$. IOP is the main risk factor for the development and progression of glaucoma [20], and IOP measurement is an important test to diagnose and monitor glaucoma. In addition, the decrease in CCT may account for IOP underestimation, which may represent a significant risk of delayed diagnosis of glaucoma. Since SMILE respects Bowman's layer more so than procedures using larger incisions, it might be assumed that corneal biomechanics could be less affected and that the impact on IOP decrease might therefore be lower than in other procedures with a comparable decrease in CCT.

Patients undergoing SMILE or FS-LASIK surgery are exposed to IOP variations during the surgery and the postoperative period. Our study demonstrated a drop in IOP of $5.01 \pm 2.08 \mathrm{mmHg}$ at 3 months (mean ablation depth: $81.24 \pm 24.76 \mu \mathrm{m}$ ) after surgery, and this postoperative IOP decrease was positively correlated with CCT changes. Li et al. found no difference between SMILE and FS-LASIK surgeries in postoperative IOP values or in IOP decrease as a function of ablation thickness (SMILE vs. FS-LASIK, $0.05 \pm 0.02$ vs. $0.05 \pm 0.03 \mathrm{mmHg} / \mu \mathrm{m})$ using NCT [15]. Our study showed a 
TABLE 1: Clinical parameters of study subjects.

\begin{tabular}{lccc}
\hline Parameters & Preoperative $($ mean \pm SD) & 3 months postoperatively $($ mean \pm SD) & $P$ \\
\hline Mean age (years) & $29.42 \pm 4.47$ & NA & NA \\
Programmed ablation depth $(\mu \mathrm{m})$ & $81.24 \pm 24.76$ & NA & NA \\
CCT $(\mu \mathrm{m})$ & $545.98 \pm 26.61$ & $0.40 \pm 30.26$ & $P<0.01$ \\
Spherical equivalent refraction $(D)$ & $-3.15 \pm 1.50$ & $24.70 \pm 0.28$ & $P<0.01$ \\
Axial length $(\mathrm{mm})$ & $24.80 \pm 0.84$ & $P<0.01$ \\
\hline
\end{tabular}

Data are presented as mean \pm SD.

TABLE 2: Mean peripapillary RNFL thickness $(\mu \mathrm{m})$, RNFL thickness in 4 quadrants of optic papilla, mean macular GCC thickness and minimum macular GCC thickness before and 3 months after SMILE surgery.

\begin{tabular}{lccr}
\hline Parameters & Preoperative $($ mean \pm SD) & 3 months postoperatively $($ mean \pm SD) & $P$ \\
\hline RNFL avg & $94.42 \pm 8.26$ & $95.16 \pm 8.50$ & $P>0.05$ \\
RNFL T & $70.54 \pm 17.33$ & $70.80 \pm 18.42$ & $P>0.05$ \\
RNFL N & $70.94 \pm 11.85$ & $69.44 \pm 12.53$ & $P>0.05$ \\
RNFL I & $121.0 \pm 14.71$ & $120.5 \pm 20.51$ & $P>0.05$ \\
RNFL S & $114.7 \pm 11.92$ & $117.7 \pm 13.67$ & $P>0.05$ \\
GCC avg & $78.08 \pm 11.00$ & $79.78 \pm 6.22$ & $P>0.05$ \\
GCC min & $78.54 \pm 5.08$ & $79.22 \pm 5.14$ & $P>0.05$ \\
\hline
\end{tabular}

RNFL avg, retinal nerve fiber layer average; RNFL T, retinal nerve fiber layer in temporal quadrant; RNFL N, retinal nerve fiber layer in nasal quadrant; RNFL I, retinal nerve fiber layer in inferior quadrant; RNFL S, retinal nerve fiber layer in superior quadrant; GCC avg, GC-IPL ganglion cell layer + inner plexiform layer average thickness; GCC min, GC-IPL ganglion cell layer + inner plexiform layer minimal thickness. Data are presented as mean \pm SD.

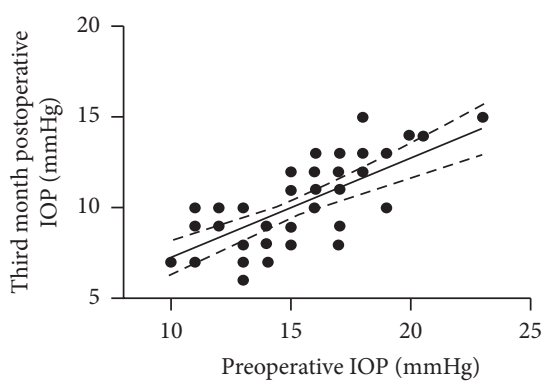

(a)

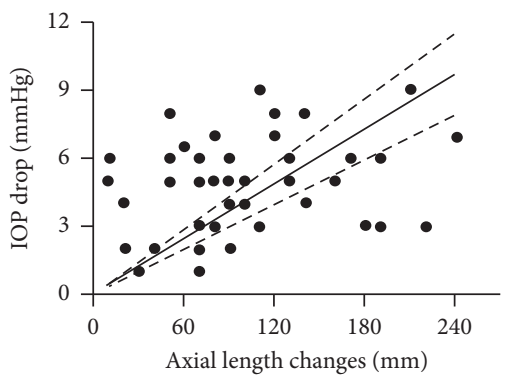

(d)

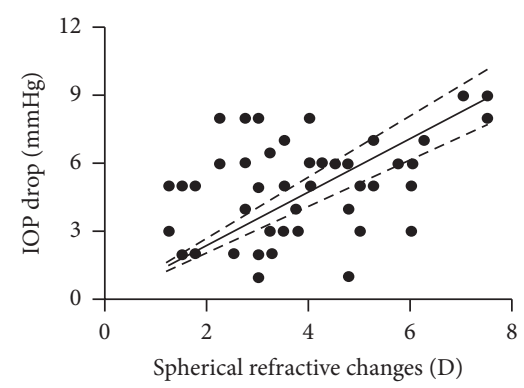

(b)

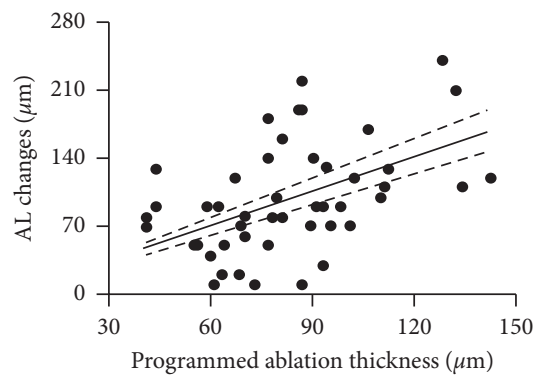

(e)

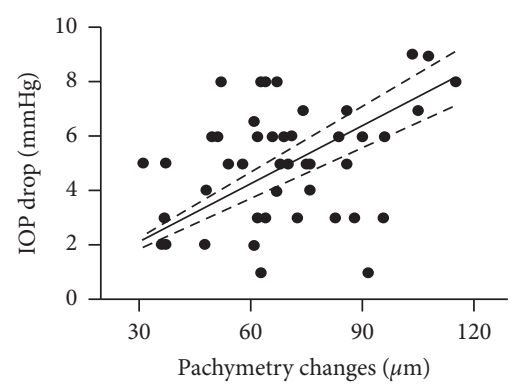

(c)

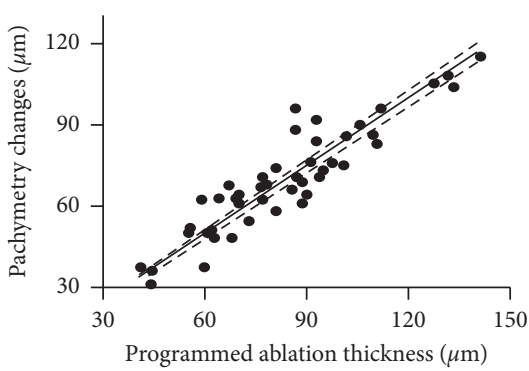

(f)

Figure 2: (a) Postoperative IOP being positively correlated with preoperative IOP values $\left(R^{2}=0.46, P<0.01\right)$. (b) The decrease in IOP being correlated with preoperative spherical refraction $\left(R^{2}=0.1083, P>0.05\right)$. (c) Correlation analysis between IOP decrease and pachymetry changes $\left(R^{2}=0.1231, P>0.05\right)$. (d) Correlation analysis between IOP decrease and axial length changes $\left(R^{2}=0.04032, P>0.05\right)$. (e) Correlation analysis between programmed ablation thickness and axial length changes $\left(R^{2}=0.1696, P<0.01\right)$. (f) Correlation analysis between programmed ablation thickness and pachymetry changes $\left(R^{2}=0.9309, P<0.01\right)$.

similar pre- and postoperative difference of $0.065 \pm 0.031 \mathrm{mmHg} / \mu \mathrm{m}$ as a function of ablation thickness. However, SMILE requires a slightly thicker corneal ablation depth using the VisuMax system [16] to correct the same refraction as compared to PRK and LASIK using the Munnerlyn formula [21]. For example, LASIK removes
$48 \mu \mathrm{m}$ to correct $-4 \mathrm{D}$, while SMILE creates a lenticular depth of $81 \mu \mathrm{m}$ to correct the same amount of myopia. Our results for postoperative drop in IOP per diopter after SMILE $(1.187 \mathrm{mmHg} / \mathrm{D})$ were higher than those reported after LASIK or PRK (about $0.5 \mathrm{mmHg} / \mathrm{D}$ ) [22], suggesting that SMILE probably results in a lower postoperative IOP 
than LASIK or PRK. Studies have demonstrated that SMILE might better preserve the anterior residual stroma than PRK or LASIK, which means a better postoperative total corneal tensile strength $[16,23]$ after SMILE. Nevertheless, this difference in anterior corneal architecture after surgery might not impact the measurement of IOP using NCT.

Glaucoma is generally described as a loss of retinal ganglion cell axons, demonstrated by the thinning of the peripapillary RNFL and GCC complex. In particular, GCC now serves as an early marker of glaucoma progression, as it contains the ganglion cell axons, cell bodies, and dendrites, which are preferentially affected by elevated IOP. Over $50 \%$ of the ganglion cells are localized [24] to the macular region, including the ganglion cell layer and inner plexiform layer. The macular GCL thickness was therefore measured in our study to represent the GCC complex. We found that neither average peripapillary RNFL thickness nor average macular GCL thickness changed at 3 months in spite of the acute IOP spike during the SMILE procedure. Although postoperative minimum GC-IPL thickness was greater than the preoperative data, the difference was only in the order of $0.7 \mu \mathrm{m}$, which is within the minimal resolution of OCT scans. Some authors [12] have reported minor early-postoperative variations of RNFL thickness after SMILE surgery which was below the axial resolution limit of optical coherence tomography devices. Our results are consistent with previous studies on SMILE [12], laser-assisted subepithelial keratomileusis (LASEK) [25], FS-LASIK [26], and PRK [27] showing the absence of postoperative effects on RNFL and GCL thickness.

Axial length is essential for intraocular lens (IOL) power calculation in lens-based surgery [28]. With the increasing number of patients undergoing laser refractive surgery, a predictable and accurate IOL selection is more challenging in these patients than in those without prior refractive surgery. As for patients having undergone FS-LASIK or PRK surgery, various formulas such as OCT-based intraocular lens power formula, the Barrett True- $\mathrm{K}$ formula, and the formulas on the American Society of Cataract and Refractive Surgery (ASCRS) calculator [29] have been proposed for their accurate IOL power calculation. The IOLMaster is a noncontact device that measures the length from the corneal surface to the retinal pigment epithelium along the visual axis using partial coherence interferometry. It measures the delay and intensity of reflected infrared laser light to determine distances, which is shown to be faster and more accurate than traditional ultrasonic pachymetry [30,31]. We chose to measure the axial length at 3 months after surgery to allow any stromal bed edema resulting from surgical manipulation to resolve. Our results demonstrated decreased AL values after SMILE surgeries, which were correlated with programmed ablation thickness. An accurate re-evaluation of AL should thus be performed before cataract surgery among post-SMILE patients.

SMILE surgery thus modified IOP, CCT, and AL measurements. A larger sample size of patients would be needed to evaluate the IOP reduction based on preoperative IOP baseline and refractive errors. Further studies using different IOP measurement methods along with postoperative corneal biomechanical analysis in comparison with LASIK/PRK would help to better understand the effects of corneal structural changes on IOP after refractive surgeries.

\section{Data Availability}

The data used to support the findings of this study are available from the corresponding author upon request.

\section{Conflicts of Interest}

The authors declare that there are no conflicts of interest.

\section{References}

[1] K. Kamiya, K. Shimizu, A. Igarashi, and H. Kobashi, "Visual and refractive outcomes of femtosecond lenticule extraction and small-incision lenticule extraction for myopia," American Journal of Ophthalmology, vol. 157, no. 1, pp. 128-134, 2014.

[2] A. Ivarsen, S. Asp, and J. Hjortdal, "Safety and complications of more than 1500 small-incision lenticule extraction procedures," Ophthalmology, vol. 121, no. 4, pp. 822-828, 2014.

[3] D. Wu, Y. Wang, L. Zhang, S. Wei, and X. Tang, "Corneal biomechanical effects: small-incision lenticule extraction versus femtosecond laser-assisted laser in situ keratomileusis," Journal of Cataract \& Refractive Surgery, vol. 40, no. 6, pp. 954-962, 2014.

[4] M. Blum, K. Täubig, C. Gruhn, W. Sekundo, and K. S. Kunert, "Five-year results of small incision lenticule extraction (ReLEx SMILE)," British Journal of Ophthalmology, vol. 100, no. 9, pp. 1192-1195, 2016.

[5] J. L. Hernández-Verdejo, L. D. Benito-Llopis, and M. A. Teus, "Comparison of real-time intraocular pressure during laser in situ keratomileusis and epithelial laser in situ keratomileusis in porcine eyes," Journal of Cataract \& Refractive Surgery, vol. 36, no. 3, pp. 477-482, 2010.

[6] J. M. Vetter, A. Schirra, D. Garcia-Bardon, K. Lorenz, W. E. Weingärtner, and W. Sekundo, "Comparison of intraocular pressure during corneal flap preparation between a femtosecond laser and a mechanical microkeratome in porcine eyes," Cornea, vol. 30, no. 10, pp. 1150-1154, 2011.

[7] W. Cheng, L. Liu, S. Yu et al., "Real-time intraocular pressure measurements in the vitreous chamber of rabbit eyes during small incision lenticule extraction (SMILE)," Current Eye Research, vol. 43, no. 10, pp. 1260-1266, 2018.

[8] M. Ang, S. S. Chaurasia, R. I. Angunawela et al., "Femtosecond lenticule extraction (FLEx): clinical results, interface evaluation, and intraocular pressure variation," Investigative Ophthalmology \& Visual Science, vol. 53, no. 3, pp. 1414-1421, 2012.

[9] J. F. Arevalo, F. J. RodriguezJ. L. Rosales-Meneses et al., Vitreoretinal surgery for macular hole after laser assisted in situ keratomileusis for the correction of myopia," British Journal of Ophthalmology, vol. 89, no. 11, pp. 1423-1426, 2005.

[10] B. Qin, L. Huang, J. Zeng, and J. Hu, "Retinal detachment after laser in situ keratomileusis in myopic eyes," American Journal of Ophthalmology, vol. 144, no. 6, pp. 921-923, 2007.

[11] J. Zhang and Y. H. Zhou, "Effect of suction on macular thickness and retinal nerve fiber layer thickness during LASIK used femtosecond laser and Moria M2 microkeratome," International Journal of Ophthalmology, vol. 8, no. 4, pp. 777783, 2015. 
[12] J. Zhang, Y. Zhou, Y. Zheng, Q. Liu, C. Zhai, and Y. Wang, "Effect of suction on macular and retinal nerve fiber layer thickness during femtosecond lenticule extraction and femtosecond laser-assisted laser in situ keratomileusis," Journal of Cataract \& Refractive Surgery, vol. 40, no. 12, pp. 1994-2001, 2014.

[13] L. Feng, F. Lei, S. A. Burns, L. Shao, and Y Yang, "Retinal measurements using time domain OCT imaging before and after myopic lasik," Ophthalmic \& Physiological Optics: The Journal of the British College of Ophthalmic Opticians (Optometrists), vol. 32, no. 3, pp. 222-227, 2012.

[14] J. S. Titiyal, M. Kaur, F. Shaikh, M. Gagrani, A. Brar, and A. Rathi, "Small incision lenticule extraction (SMILE) techniques: patient selection and perspectives," Clinical Ophthalmology, vol. 12, pp. 1685-1699, 2018.

[15] H. Li, Y. Wang, R. Dou et al., "Intraocular pressure changes and relationship with corneal biomechanics after smile and FS-LASIK," Investigative Opthalmology \& Visual Science, vol. 57, no. 10, pp. 4180-4186, 2016.

[16] D. Z. Reinstein, T. J. Archer, and M. Gobbe, "Small incision lenticule extraction (SMILE) history, fundamentals of a new refractive surgery technique and clinical outcomes," Eye and Vision, vol. 1, no. 1, 2014.

[17] Y.-C. Tham, X. Li, T. Y. Wong, H. A. Quigley, T. Aung, and C.-Y. Cheng, "Global prevalence of glaucoma and projections of glaucoma burden through 2040," Ophthalmology, vol. 121, no. 11, pp. 2081-2090, 2014.

[18] M. W. Marcus, M. M. De Vries, F. G. J. Montolio, and N. M. Jansonius, "Myopia as a risk factor for open-angle glaucoma: a systematic review and meta-analysis," Ophthalmology, vol. 118, no. 10, pp. 1989-1994, 2011.

[19] S. J. Chen, P. Lu, W. F. Zhang, and J. H. Lu, "High myopia as a risk factor in primary open angle glaucoma," International Journal of Ophthalmology, vol. 5, no. 6, pp. 750-753, 2012.

[20] M. O. Gordon, J. A. Beiser, J. D. Brandt et al., "The ocular hypertension treatment study," Archives of Ophthalmology, vol. 120, no. 6, pp. 714-712, 2002.

[21] A. W. Chang, A. C. Tsang, J. E. Contreras et al., "Corneal tissue ablation depth and the Munnerlyn formula," Journal of Cataract \& Refractive Surgery, vol. 29, no. 6, pp. 1204-1210, 2003.

[22] R. Crnic-Rein and W. Neil Charman, "Intraocular pressure after excimer laser myopic refractive surgery," Ophthalmic and Physiological Optics, vol. 21, no. 3, pp. 228-235, 2001.

[23] Y. Shen, X. Su, X. Liu, H. Miao, X. Fang, and X. Zhou, "Changes in intraocular pressure values measured with noncontact tonometer (NCT), ocular response analyzer (ORA) and corvis scheimpflug technology tonometer (CST) in the early phase after small incision lenticule extraction (SMILE)," BMC Ophthalmology, vol. 16, no. 1, 205 pages, 2016.

[24] Y. J. Kim, M. H. Kang, H. Y. Cho, H. W. Lim, and M. Seong, "Comparative study of macular ganglion cell complex thickness measured by spectral-domain optical coherence tomography in healthy eyes, eyes with preperimetric glaucoma, and eyes with early glaucoma," Japanese Journal of Ophthalmology, vol. 58, no. 3, pp. 244-251, 2014.

[25] N. Sharma, P. Sony, A. Gupta, and R. B. Vajpayee, "Effect of laser in situ keratomileusis and laser-assisted subepithelial keratectomy on retinal nerve fiber layer thickness," Journal of Cataract \& Refractive Surgery, vol. 32, no. 3, pp. 446-450, 2006.

[26] L. M. Zangwill, T. Abunto, C. Bowd, R. Angeles, D. J. Schanzlin, and R. N. Weinreb, "Scanning laser polarimetry retinal nerve fiber layer thickness measurements after LASIK," Ophthalmology, vol. 112, no. 2, pp. 200-207, 2005.

[27] A. P. Aristeidou, G. Labiris, E. I. Paschalis, N. C. Foudoulakis, S. C. Koukoula, and V. P. Kozobolis, "Evaluation of the retinal nerve fiber layer measurements, after photorefractive keratectomy and laser in situ keratomileusis, using scanning laser polarimetry (GDX VCC)," Graefe's Archive for Clinical and Experimental Ophthalmology, vol. 248, no. 5, pp. 731-736, 2010.

[28] R. B. Melles, J. T. Holladay, and W. J. Chang, "Accuracy of intraocular lens calculation formulas," Ophthalmology, vol. 125, no. 2, pp. 169-178, 2017.

[29] L. Wang, M. Tang, D. Huang, M. P. Weikert, and D. D. Koch, "Comparison of newer intraocular lens power calculation methods for eyes after corneal refractive surgery," Ophthalmology, vol. 122, no. 12, pp. 2443-2449, 2015.

[30] O. Findl, W. Drexler, R. Menapace, C. K. Hitzenberger, and A. F. Fercher, "High precision biometry of pseudophakic eyes using partial coherence interferometry," Journal of Cataract \& Refractive Surgery, vol. 24, no. 8, pp. 1087-1093, 1998.

[31] A. B. Bhatt, A. C. Schefler, W. J. Feuer et al., "Comparison of predictions made by the intraocular lens master and ultrasound biometry," Archives of Ophthalmology, vol. 126, no. 7, pp. 929-933, 2008. 